\title{
Tuning diazonium modification of gold nanoparticles for fuel cell application
}

\author{
Atieh Aghajani ${ }^{1}$, Marie-Pierre Santoni ${ }^{1}$, Peyman Mirzaei $^{2}$, \\ Ahmed A. Mohamed ${ }^{3}$, Mohamed M. Chehimi ${ }^{1,2, *}$ and Mohamed Jouini ${ }^{1, *}$ \\ ${ }^{1}$ Université de Paris, ITODYS, UMR CNRS 7086, 15 rue JA de Baïf, 75013 Paris, France \\ ${ }^{2}$ Université Paris Est, CNRS, ICMPE (UMR 7182), 94320 Thiais, France \\ ${ }^{3}$ Center for Advanced Materials Research, Research Institute of Sciences and Engineering, \\ University of Sharjah, Sharjah 27272, UAE
}

\begin{abstract}
Products made from alcohol oxidation are very important and used in food industry, pharmaceuticals and dryers to name but a few. In this regard, precious metals such as gold operate in various shapes and sizes as effective catalysts for the oxidation of alcohols. In this work, $20 \mathrm{~nm}$-sized gold nanoparticles were synthesized and modified with either 4carboxybenzenediazonium tetrafluoroborate $(\mathrm{AB})$ (single salt catalyst) or a mixture of the latter with 3,5-dimethylbenzenediazonium tetrafluoroborate (AB-DMB, double-salt catalyst). The relative concentrations of the diazonium salts were tuned in order to maintain the electrocatalytic property of the arylated gold nanoparticles in the electro-oxidation of ethanol. The physicochemical properties (size, aryl layer thickness and composition) and electrocatalytic performances of the capped gold nanoparticles (in single and double salt catalyst systems) were investigated by means of TEM, XPS, UV-visible, TGA-DTA and electrochemistry. The aryl capping layer thickness is below $1 \mathrm{~nm}$ when the total salt concentration was $5 \times 10^{-5} \mathrm{M}$, without any significant loss of current compared to pure gold nanoparticles. For $1 \mu \mathrm{L}$ of pristine or arylated Au NPs, coated on $0.07 \mathrm{~cm}^{2}$ glassy carbon electrode disk, we achieved a low oxidation potential of $200 \mathrm{mV}$ and high current ethanol oxidation peak intensity levelling off at $\sim 80 \mu \mathrm{A}$ for the single diazonium arylation. It is demonstrated that dilution of the carboxylic acid-functionalized diazonium is more important than mixing this compound with 3,5-dimethylbenzene diazonium salt at equal total concentration. The dual arylation strategy returned lower ethanol oxidation intensity likely due to hydrophic effect imparted by the 3,5-dimethylphenyl groups, thus hindring access of ethanol to the nanocatalyst surface.

This work demonstrates that arylated gold nanoparticles are unique nanocatalysts for ethanol oxidation reaction, with remarkable performances. This electrocatalytic performance of the nanoparticles was maintained through the use of finely tuned aryl capping layer. The strategy opens avenues for other arylated electrocatalysts and uses thereof.
\end{abstract}

Keywords: gold nanoparticles, diazonium salts, electrocatalyst, electro-oxidation, ethanol, fuel cell.

Correspondence: Mohamed M. Chehimi, UP \& CNRS (UMR 7086), mmchehimi@ yahoo.fr 


\section{Graphical abstract}

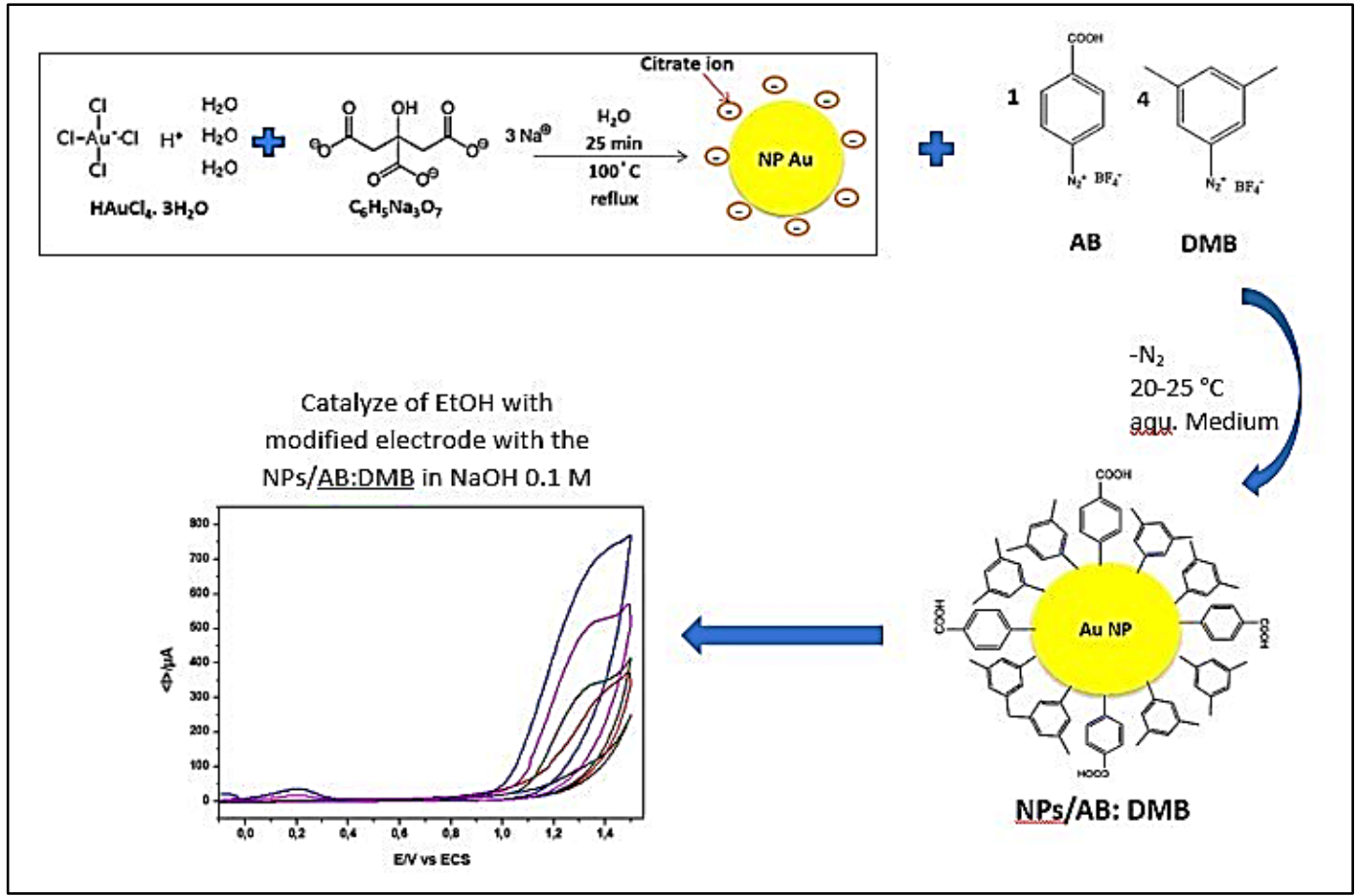

\section{Highlights}

- $20 \mathrm{~nm}$-sized gold nanoparticles were prepared and arylated with single or diazonium salt mixtures.

- Arylated Au nanoparticles served as electrocatalysts of ethanol oxidation which occurred at $200 \mathrm{mV}$ vs $\mathrm{Ag} / \mathrm{AgCl}$.

- Single arylation of Au NPs with carboxyphenyl groups from $5 \times 10^{-5} \mathrm{M}$ single diazonium solution yielded high ethanol oxidation current.

- Tuned arylation of Au NPs ensures dispersion and adhesion without compromising electrocatalytic activity 


\section{Introduction}

Hybrid materials combine the best of the salient features of their organic and inorganic counterparts, and synergetically exhibit outstanding new properties [1-4]. Hybrids are employed with success in numerous domains such as nanobiomedicine [5-7], analytical sciences [8], catalysis [9, 10], electrochemistry [11, 12], environmental remediation [13, 14], energy sector [15], electronics and optoelectronics [16], and forensics [17] to name but a few.

In hybrid systems, organic components could ensure a particular (re)activity, whereas the inorganic constituents allow multi-electronic redox activity, of importance in catalysis [18]. The synergy of the properties of the organic and inorganic counterparts induces performances not achievable by the counterparts taken separately, hence the utilization of hybrid materials in various domains.

Particularly, metallic nanoparticles (NPs) and their hybrid systems are the subject of numerous studies pertaining to the development of novel specialty electrodes used in photovoltaic cells (conversion of solar energy) [19, 20], hydrogen evolution reaction (HER) [21], and numerous other catalyzed reduction and oxidation processes [22], chemical or electrochemical sensors (specific and selective detection) [23] or materials with specific surface properties (e.g. biological anti-adhesion, lubrication, photo- or thermo-sensitive layer) [24]. With the rapid development of fuel cells, ethanol oxidation reaction (EOR) has received much attention due to the lower toxicity of this alcohol compared to methanol. EOR requires electrocatalysts to achieve acceptable performances. In the recent years, it became clear that making hybrid nanocatalysts is an elegant route for high performance EOR-based systems. In this regard, for example, Pandey et al. [25] used organic-mineral hybrid materials containing gold nanoparticles using different organic conducting polymers for ethanol $(\mathrm{EtOH})$ oxidation. In the same way, after deposition of an evaporated gold film, they deposited various polymers using the galvanostatic method to obtain a hybrid film. They also explained that nanoparticles of gold in alkaline medium exhibited high catalytic activity for ethanol oxidation. In this regard, there are other studies that show that gold has an excellent electrocatalytic property for the electro-oxidation of alcohols, as well as the reduction of protons to hydrogen [26-29].

For oxidation of ethanol, it is also possible to use other noble metals such as palladium $(\mathrm{Pd})$ and platinum $(\mathrm{Pt})$ due to the fact that they have electrocatalytic properties in different electrolytes and $\mathrm{pH}[30,31]$. However, the scarcity and high cost of these metals have led to the development of new structures and electrode materials; for example, using the metals in the form of nanoparticles and also use them as hybrids in order to limit the quantities of metals used while retaining the electrocatalytic properties. Gold organometallic chemistry permits to design numerous gold-based nanosystems; for example through arylation [4]. In this regard, Laurentius et al. [32] and Mirkhalaf et al. [33] employed diazonium salts for enhancing dispersion of gold nanoparticles and/or attachment to electrode surfaces. Arylation of the nanoparticles is tricky because it should be done in a way that charge transfer within the hybrid interface (electrode-aryl-NPs) should not be hindered. Moreover, arylation of the 
electrode or the electrode material itself has major effect on the adhesion and stability of the nanoparticles at electrode surfaces [12, 34, 35].

There are different methods for the grafting of aryl groups to metal nanoparticles and other surfaces [36]. The team of Gooding [37] obtained a hybrid layer containing gold nanoparticles and diazonium salts on the surface of the electrode. Initially, they modified the glassy carbon electrodes (GC) surface with the electrochemical method, using diazonium salts 4aminophenyl and 4-thiophenol, and then they grafted gold nanoparticles on diazonium salts grafted through covalent bonds with different captors $\mathrm{C}, \mathrm{N}$ and $\mathrm{S}$. Various characterizations indicate that the modification is well established and long term stable (in acidic medium). It is an easy, fast, and reliable technique to functionalize GC surfaces with robust attachment of AuNPs. Mirkhalaf et al. [38] showed that the bonding of arylated gold nanoparticles to surfaces can be done through the interactions between long alkyl chains borne by the latter with similar ones from decylphenyl-modified electrode surface. The nanocatalysts were thus found in a local hydrophobic environment at the gold-surface interface and could selectively electrocatalyze oxygen reduction. Gam-Derouich et al. [39] electroarylated gold flat surface for the attachment of AuNPs. The latter were post-arylated in order to trigger radical graftphotopolymerization therefore obtaining nanostructured thin polymer sensing layers of dopamine. This strategy has been extended to the robust deposition of Au and Ag NPs on agrowaste powder particles [40] and carbon nanotubes [41], $\mathrm{Rh}-\mathrm{Cu}$ bimetallic NPs to high surface area graphite powder [12], carbon nanotubes to flexible ITO electrode surface [35]. For electrocatalytic purpose, it is essential to keep the extent of arylation as low as possible to avoid passivation of the nanocatalyst and inhibition of electron transfer. Such a passivation is usually due to the formation of oligoaryl layer [36]. In order to obtain a monolayer, one could arylate the surface with aryl diazonium compounds bearing blocked positions 3 and 5 of the benzenediazonium [42]. In addition, combining such a sterically hindered diazonium salt with a diazonium compound bearing a reactive group will permit to modify surfaces with aryl diazonium mixtures, an elegant way to tune surface arylation for targeted applications [43].

This work aims at investigating and comparing the electrocatalytic properties of gold nanoparticles before and after grafting aryl groups from one or two types of diazonium salts in the oxidation of ethanol. In addition, the initial diazonium salt concentrations were step wisely monitored in order to tune the composition and grafting density of the surface bound organic layer while maintaining the catalytic properties of gold nanoparticles as well as their stability, therefore preventing their aggregation (maintaining a higher level of active nanoparticles). Gold NPs of controlled size in aqueous solution were prepared and stabilized with citrate salts via the Turkevich-Frens method [44]. We have functionalized NPs through the reductive grafting reaction of diazonium salts, focusing on the tuned attachment of carboxyphenyl groups. The main challenge of this work was to control the number of acid functions introduced, without compromising the catalytic properties of gold NPs via excessive arylation. To address this issue, mixtures of para-carboxybenzenediazonium and 3,5dimethylbenzenediazonium salts were used. The former serves as a basis for coupling with other functional groups for hybrid materials with a variety of applications, while the latter is used to hamper any possible oligomerization and growth of thick aryl that passivates the surface of the nanocatalyst [45]. Different concentrations of diazonium salts AB and DMB 
were investigated to obtain optimal conditions for ethanol oxidation. Catalytic activity of the pristine and diazonium-modified NPs vis-à-vis ethanol oxidation reaction was assessed by cyclic voltammetry.

\section{Experimental}

\subsection{Reagents materials and instruments}

Solvents: pure ethanol (VWR, 100\%) and diethylether (VWR, 100\%). The starting materials for synthesis or for electrochemical or other studies: chloroauric acid $\left(\mathrm{HAuCl}_{4} \cdot 3 \mathrm{H}_{2} \mathrm{O}\right.$, Sigma Aldrich, 99.9\%), trisodium citrate $\left(\mathrm{C}_{6} \mathrm{H}_{5} \mathrm{Na}_{3} \mathrm{O}_{7}\right.$, Sigma Aldrich, $\left.\geq 99 \%\right)$, 4-Aminobenzoic acide (Fluka, $\geq 99 \%$ ), 3,5-dimethyl aniline (Sigma Aldrich, 98\%), sodium nitrite $\left(\mathrm{NaNO}_{2}\right.$, Prolabo, 98\%), tetrafluoroboric acid $\left(\mathrm{HBF}_{4}\right.$, Sigma Aldrich). Deionized water was used for all experiments.

Thermogravimetric analysis (TGA) and differential thermal analysis (DTA) were carried out using a Setaram Labsys Evo under pure $\mathrm{O}_{2}$. The samples were heated in a crucible alumina based with a large volume $1000 \mu \mathrm{l}$ and in the $1150 \mathrm{C}^{\circ}-1600{ }^{\circ} \mathrm{C}$ range with the weighting range +/- $1000 \mathrm{mg}$ to $+/-200 \mathrm{mg}$.

NMR spectra were recorded using a Bruker AVANCE III 400MHz spectrometer. Chemical changes are given in parts per million (ppm, d) relative to the solvent peak as the internal standard (DMSO).

X-ray photoelectron spectroscopy (XPS) analyses were performed using a K Alpha machine equipped with microfocused, monochromatic Al Ka X-ray source ( $\mathrm{h} v=1486.6 \mathrm{eV}$, spot size $=400 \mu \mathrm{m})$. The pass energy was set at 200 and $80 \mathrm{eV}$ for the survey and the narrow regions, respectively. A flood gun was used for static charge compensation. Surface chemical composition was determined using the manufacturer's sensitivity factors.

Infrared (IR) spectra were recorded with a Nicolet 8700 spectrometer with a Mercury Cadmium Telluride (MCT) detector.

TEM imaging was done with Jeol 2010 (LaB6) (Jeol Ltd., Tokyo, Japan), dedicated to the medium-resolution structural and chemical analysis. The microscope was operating at $80 \mathrm{kV}$ acceleration voltage.

UV-vis spectra were recorded on a Cary $4000 \mathrm{UV}$-Visible spectrophotometer to collect information on NP surface plasmon resonance (SPR) and their sizes.

Electrochemical properties were investigated using VSP Potentiostat Bio-Logic, with current ranging from $10 \mu \mathrm{A}$ to $1 \mathrm{~A}$. In this regard it was used from a classical three-electrode cell with a glassy carbon disk electrode (geometric area $=0.07 \mathrm{~cm}^{2}$ ) as the working electrode. The counter electrode was platinum wire and the reference electrode was $\mathrm{Ag} / \mathrm{AgCl}(3.5 \mathrm{M} \mathrm{KCl})$. Electrochemical measurements were performed in the aqueous electrolyte of $10^{-1} \mathrm{~mol} \mathrm{~L}^{-1}$ $\mathrm{NaOH}$, using software EC-Lab® Express. 


\subsection{Synthesis, purification and lyophilization of gold nanoparticles}

\subsubsection{Synthesis}

The gold salt solution $\left(\mathrm{HAuCl}_{4} \cdot 3 \mathrm{H}_{2} \mathrm{O}\right)$ was prepared at a concentration of $1 \mathrm{mM}$ after the sodium citrate reducing solution $\left(\mathrm{C}_{6} \mathrm{H}_{5} \mathrm{Na}_{3} \mathrm{O}_{7}\right)$ was added with vigorous stirring and heated to $100{ }^{\circ} \mathrm{C}$. under reflux. The color of the solution changed from light yellow, dark black, and finally to burgundy red which indicates the formation of gold nanoparticles. The boiling and stirring were maintained for $25 \mathrm{~min}$, and then the medium was left to cool to room temperature under stirring. Citrates play both the role of reducing agent and stabilizer for NPs, due to their negative charge which prevents agglomeration of particles by electrostatic repulsion.

\subsubsection{NPs suspension dialysis}

The NPs solution contains the sodium citrate as a stabilizer. These citrates are around NPs and contain $\mathrm{COO}^{-}$carboxylate functions which can disturb the XPS analysis of NPs grafted with dizonium salts contain $\mathrm{COOH}$. In this regard, we removed the citrates from the NPs solution by the dialysis method using semi-permeable exchange cellulose membranes. The time required for optimal purification varies from 6 hours to 7 days. In our case the optimal purification time is three days ( 72 hours) considering the UV analysis. After this time the solution which was red and clear became a dark blue suspension with particles visible to the naked eye, that it shows the removal of citrates from the suspension and the aggregation of NPs.

Due to the reversibility and disaggregation of these nanoparticles in solutions compatible with functional groups (hydrophilic and hydrophobic), this method can be a practical and effective method to store nanoparticles in solid state no grafted or grafted with various diazonium salts. For use in various hydrophilic and hydrophobic media, according to the ultimate goal of using these nanoparticles.

\subsubsection{Centrifugation of the suspension of grafted NPs after the dialysis step}

Subsequently we centrifuged the solution 3 times at the speed of $6000 \mathrm{rpm}$ for $30 \mathrm{~min}$ in the glass tubes. Each time the NPs precipitated at the bottom of the tubes and the discolored supernatant is removed and again filled with Milli-Q. So by centrifugation we can wash the rest of the impurities and citrates, and prepare for the next step.

\subsubsection{Lyophilization of the grafted NPs solution}

Grafted NPs solution was subjected to washing/centrifugation cycles prior to nitrogen and vacuum drying. Lyophilization permitted to remove water in order to obtain clean solid powder for analysis by XPS and TGA/DTA.

\subsection{Synthesis and proton NMR characterization of diazonium salts}

4-aminobenzoic acid (ABA) (0.2 g) and 3,5-dimethylaniline (DMA) (0.3 g) as the starting product is solubilized in $\mathrm{HBF}_{4}$ acid $(1.8 \mathrm{~mL}$ for ABA and $2 \mathrm{~mL}$ for DMA). The mixture was kept under stirring for $15 \mathrm{~min}$. After cooling the solutions to $0{ }^{\circ} \mathrm{C}$ with ice, the solution of 
sodium nitrite $\mathrm{NaNO}_{2}(0.194 \mathrm{~g}$ for ABA and $0.207 \mathrm{~g}$ for DMA $)$ as an oxidant is dissolved in a minimum of water was added dropwise to the reaction mixture and the reaction was obtained for $30 \mathrm{~min}$ at $0{ }^{\circ} \mathrm{C}$. After stirring for 30 minutes, the final product is washed with cold diethyl ether, after several washes under vacuum, a precipitate appears. The synthesized diazonium salts 4-carboxybenzenediazonium (AB) and 3-5 dimethyl diazenylbenzoic (DMB) were stored in the freezer.

For first diazonium salt, we have carried out the ${ }^{1} \mathrm{H}$ NMR spectrum of the amine starting material (ABA) and the synthesized salt for comparison and to confirm the synthesis of the product, according to ${ }^{1} \mathrm{H}$ NMR analysis $(400 \mathrm{MHz}, \mathrm{DMSO}) \delta$ (ppm). For the amine product: IR (KBr): 1H NMR (400 MHz, DMSO) $\delta(\mathrm{ppm}) ; 5.86(2 \mathrm{H}, \mathrm{s}), 6.55(2 \mathrm{H}, \mathrm{d}), 7.62(2 \mathrm{H}, \mathrm{d})$, $12.14(\mathrm{H}, \mathrm{s})$. And for the product of the diazonium salt AB: 1H NMR (400 MHz, DMSO) $\delta$ (ppm); $8.42(2 \mathrm{H}, \mathrm{d}), 8.78(2 \mathrm{H}, \mathrm{d}), 14(\mathrm{H}, \mathrm{s})$.

The comparison of the results of the ${ }^{1} \mathrm{H}$ NMR spectra of the starting product and the final product shows that:

a) The peak at $5.86 \mathrm{ppm}$, corresponding to the amine $\mathrm{NH}_{2}$ function of the starting material (ABA), which disappeared in the synthesized diazonium salt.

b) There is a shift of the aromatic proton peaks (around $2 \mathrm{ppm}$ ) and the acid peak that shows the diazonium salt is well synthesized with the $\mathrm{N}_{2}{ }^{+}$attractor group. These observations confirm the production of the product of 4-Diazenylbenzoic acid diazonium salt.

For second salt; 1H NMR (400 MHz, DMSO) $\delta(\mathrm{ppm}) ; 2.53(6 \mathrm{H}, \mathrm{s}), 5.17(2 \mathrm{H}, \mathrm{s}), 6.57(2 \mathrm{H}$, s), $6.62(\mathrm{H}, \mathrm{s})$. And for the diazonium salt synthesized the $\mathrm{NH}_{2}$ peak is gone and the other peak is shifted to the left: 1H NMR (400 MHz, DMSO) $\delta(\mathrm{ppm}) ; 2.46(6 \mathrm{H}, \mathrm{s}), 7.95(2 \mathrm{H}, \mathrm{s})$, $8.32(\mathrm{H}, \mathrm{s})$.

Thus, obtaining the DMB salt is confirmed by the disappearance of the peak of the amine $\mathrm{NH}_{2}$ in the product and removal of the peaks of the aromatic protons.

\subsection{Modification of Au nanoparticles with diazonium salts}

Arylation of Au NPs with single diazonium salt (DZ) was achieved spontaneously by simple reaction of the NPs with diazonium compounds, under mechanical stirring in aqueous media. In a typical protocol: an aqueous solution of salt DZ was prepared at a set concentration in the $10^{-3}-5 \times 10^{-5} \mathrm{M}$ range, and added dropwise to the suspension of NPs. For this grafting, we prepared an aqueous mother solution of salt DZ at a concentration of $1 \mathrm{M}$ which was diluted to reach the targeted concentration of diazonium compound: $50 \mu \mathrm{L}$ of DZ solution were added to $50 \mathrm{~mL}$ of the gold NPs solution (20 nm-sized nanoparticles). The final concentration of AB salt in the reaction mixture was $10^{-3} \mathrm{M}$. For the other concentrations, we proceeded in the same way by reducing the initial concentration of $10^{-1}$ and $5 \times 10^{-2} \mathrm{M}$ to the final concentrations $10^{-4}$ and $5 \times 10^{-5} \mathrm{M}$, respectively.

The mixture was stirred for 24 hours at room temperature; then, the resulting suspension of arylated nanoparticles was washed through centrifugation using an aqueous solution of citrate (with a concentration equal to the concentration of citrate in suspension pens of 
nanoparticles). In the case of co-grafting two types of DZ salts, we have adopted the protocol described elsewhere [46, 47].

\subsection{Electrocatalyzed oxidation of ethanol}

Arylated gold NPs were coated on the surface of a glassy carbon electrode (GCE, $0.07 \mathrm{~cm}^{2}$ ) using 2 drops of $0.5 \mu \mathrm{L}(1 \mu \mathrm{L})$ of the suspension of gold NPs which was previously well homogenized and sonicated. The nanoparticle-coated GCE was dried at $35^{\circ} \mathrm{C}$.

The behavior of the modified electrode thus obtained was measured cyclic voltammetry $(\mathrm{CV})$ in a $0.1 \mathrm{M} \mathrm{NaOH}$ basic medium in a potential range between -0.1 and $1.5 \mathrm{~V}$ vs ECS. We investigated the catalytic properties of modified nanoparticles for the oxidation of ethanol at different concentrations of $0,0.5,1,1.5,2$ and $2.5 \mathrm{M}$ ethanol. A three-electrode electrochemical cell with a volume of $20 \mathrm{~mL}$ electrolyte containing the $0.1 \mathrm{M} \mathrm{NaOH}$ solution was used. The reference, the counter and the working electrodes were saturated calomel, stainless steel grid and the coated GCE $3 \mathrm{~mm}$ diameter. GCE was polished, washed with ethanol and dried with compressed air prior to each use.

\section{Results and discussion}

The rationale for using diazonium salts with hydrophilic heads was that in addition to binding to other functional groups, we could also maintain its dispersion in aqueous suspension. As aryl groups are known to form oligomers leading to a thick aryl layer and consequently passivation of the electrocatalysts, we have verified two routes: in the first one, diazonium concentration was monitored to reach a suitable thickness that ensures colloidal stability and also electrocatalytic activity. In the second approach, a refined strategy consisted in blocking the growth of the aryl layer by using two different diazonium salts; one bearing the $\mathrm{COOH}$ group and the other equipped with two methyl groups in meta positions of the diazonium salts in order to inhibit any oligomerization of the aryl groups at the surface of gold NPs. In this case, dual functionalization with two kinds of aryl groups permits to tune both colloidal stability and electrocatalytic performances of the Au NPs.

The general strategy is schematically illustrated in Figure 1. In a first step, the well-known Turkevich method [44] was adopted to design the Au NPs which were further grafted with aryl diazonium compounds; for the sake of simplicity, only the dual modification is displayed. In a third step, the GC electrodes were coated with diazonium-modified gold NPs for electrocatalyzed oxidation of ethanol. The whole strategy is simple and efficient. Hereafter, we shall describe the main experimental results pertaining to the characterization of the modified gold nanoparticles and their electrocatalytic performances. 


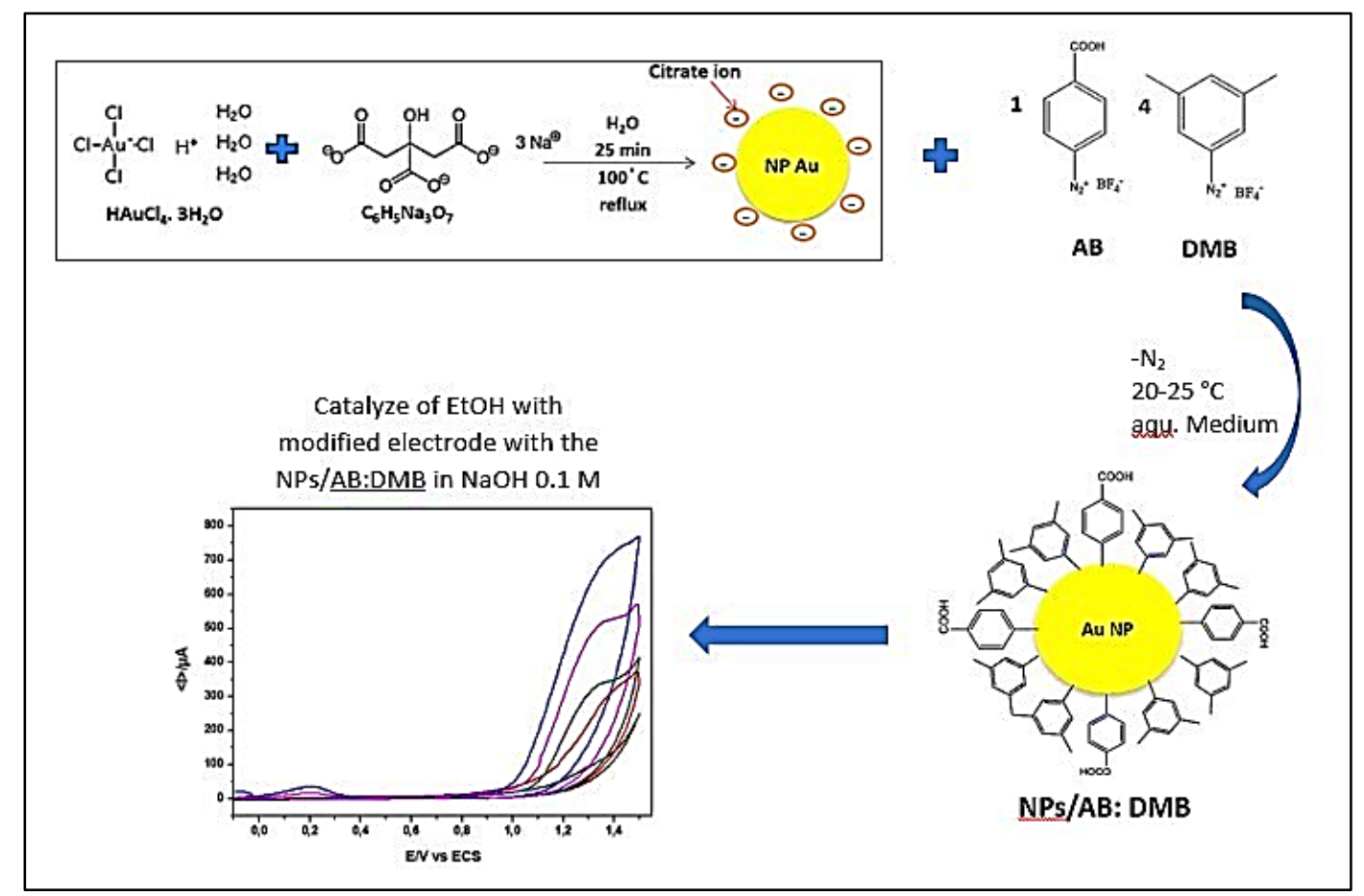

Figure 1. Strategy for the preparation of gold nanoparticles, their co-grafting with diazonium salts and their electrocatalytic performances for ethanol oxidation.

\subsection{Optical properties: UV-Visible spectra}
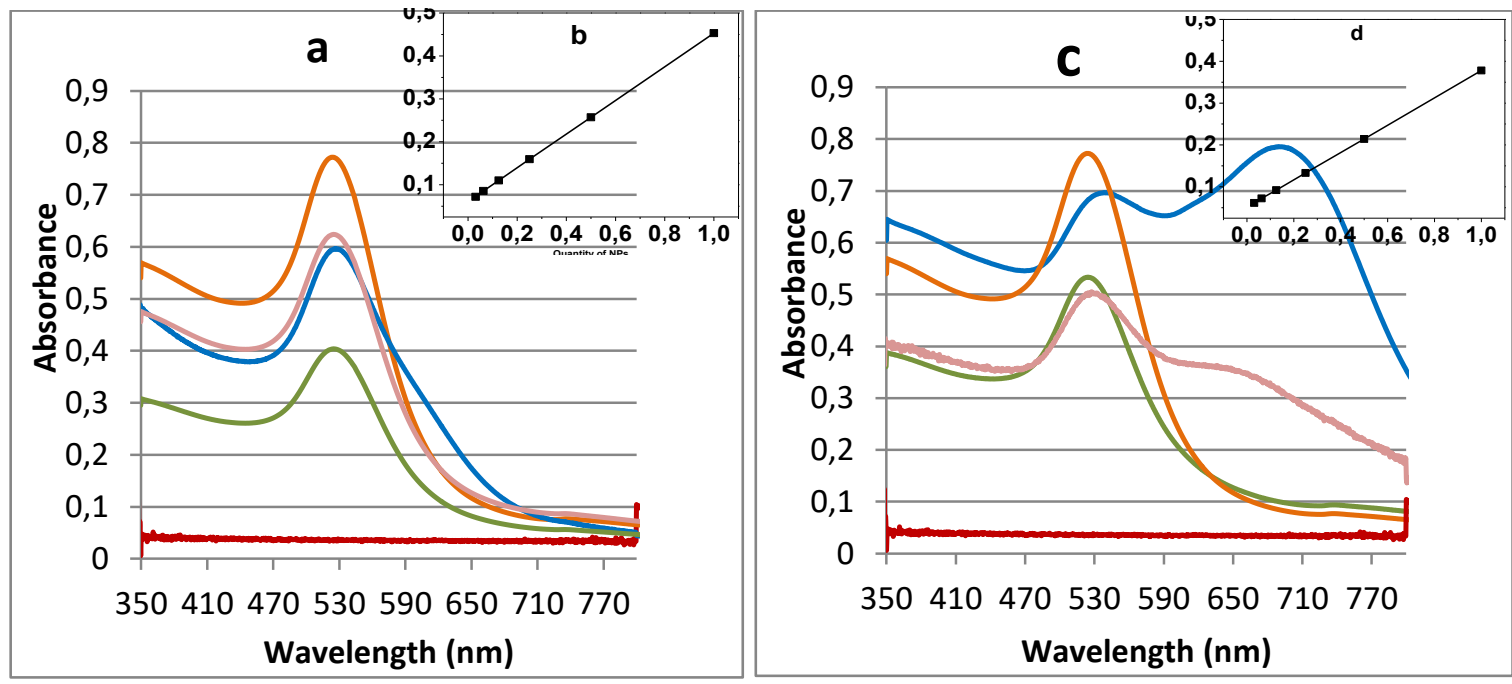

Figure 2. Comparison of the UV-Visible spectra of: a) Pristine gold NPs in orange and NPs grafted with in different concentration: $10^{-3}$ in blue, $10^{-4}$ in pink and $5 \times 10^{-5} \mathrm{M}$ in green, b) Linear curve of the absorbance of grafted NPs depending on the concentration of NPs. c) Pristine gold NPs in orange, co-grafted NPs with in different concentration: $10^{-3}$ in blue, $10^{-4}$ in pink and $5 \times 10^{-}$ ${ }^{5} \mathrm{M}$ in green, d)Linear curve of the absorbance of grafted NPs depending on the concentration of NPs. 
In Figure $2 \mathrm{a}$ it can be seen different concentration of diazonium salt $\mathrm{AB}$ from $10^{-3}$ to $5 \times 10^{-5} \mathrm{M}$ grafted on gold NPs. In the higher concentration regime, i.e. $10^{-4}$ and $10^{-3} \mathrm{M}$, the UV-Visible spectrum shows a widening of the peak in the range of 600 to $700 \mathrm{~nm}$ and the more obvious shift which shows a weak aggregation of NPs [48]. Figure 2c displays the UV-visible spectra of NPs co-grafted with $\mathrm{AB}$ and DMB (1:4) diazonium salts at indicated concentrations. This figure also shows that higher concentrations of diazonium salts induce aggregated NPs with a peak or shoulder in the high wavelength at $600-700 \mathrm{~nm}$, which will affect the catalytic activity. Figures $2 b, d$ show absorbance- $v s$-concentration linear correlations for gold NPs grafted with aryl groups (single and dual modifications) in the concentration of $5 \times 10^{-5} \mathrm{M}$.

\subsection{TEM: morphology of the nanoparticles}

The NPs were imaged using at high resolution transmission electron microscopy.

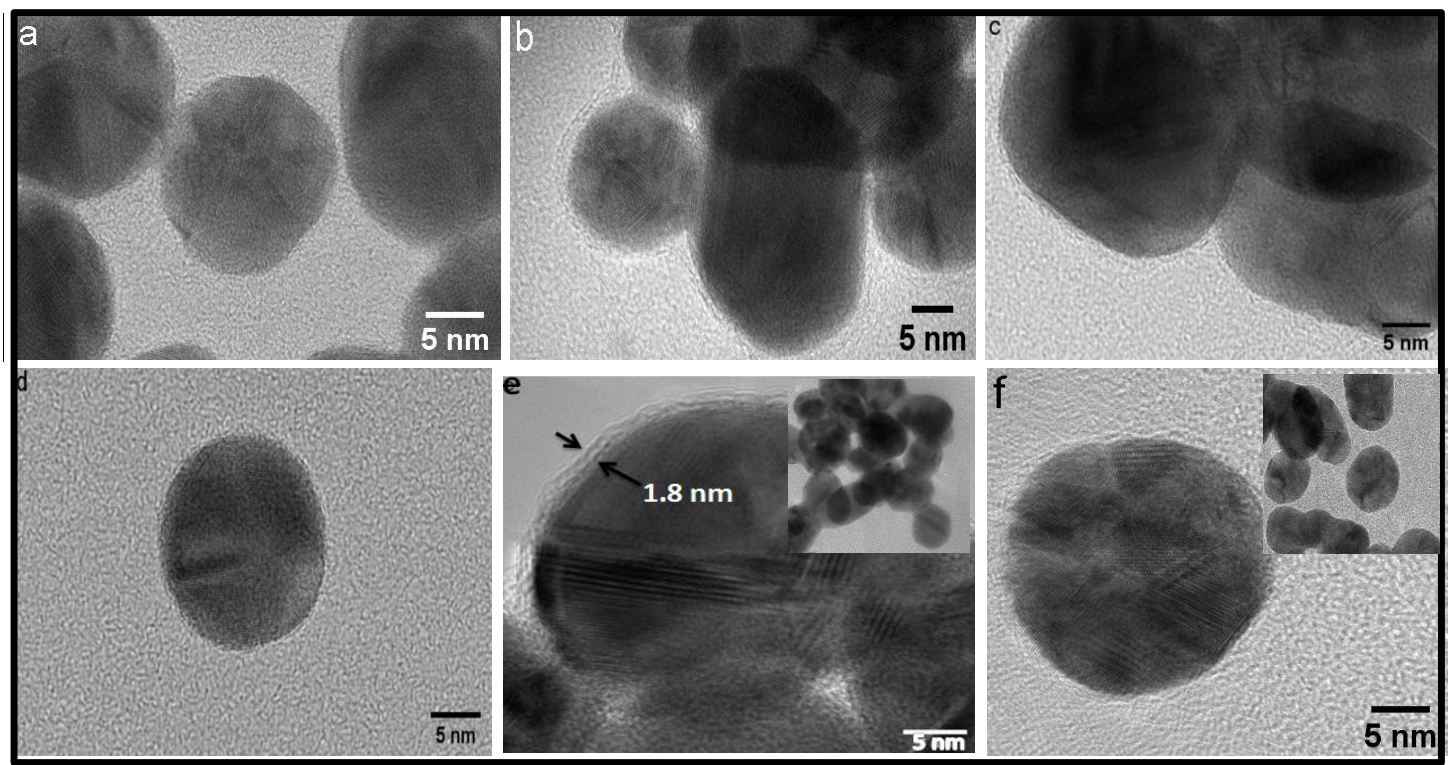

Figure 3. TEM images of a) Pristine gold NPs, b), c) and d) grafted NPs Au/AB in $10^{-3} \mathrm{M}, 10^{-4} \mathrm{M}$ and $5 \times 10^{-5} \mathrm{M}$ of salt concentrations respectively, e) and f) co-grafted NPs Au/AB:DMB $10^{-4} \mathrm{M}$ and $5 \times 10^{-5}$ $\mathrm{M}$ of salts concentrations, respectively.

Figure 3 shows TEM image of pristine (Figure 3a) and modified gold NPs with diazonium salt $\mathrm{AB}$ at concentrations of $10^{-3} \mathrm{M}, 10^{-4} \mathrm{M}$ and $5 \times 10^{-5} \mathrm{M}$ (Figure $3 \mathrm{~b}, 3 \mathrm{c}$ and $3 \mathrm{~d}$ ). TEM pictures of gold NPs modified with diazonium mixtures (total concentrations $10^{-4} \mathrm{M}$ and $5 \times 10^{-5} \mathrm{M}$ ) are displayed in Figures $3 \mathrm{e}$ and $3 \mathrm{f}$.

Compared to pristine nanoparticles, gold NPs modified with diazonium $\mathrm{AB}$ at of $10^{-3}, 10^{-4}$ and $5 \times 10^{-5} \mathrm{M}$ concentrations, the aryl layer with approximate thicknesses 2.1 and 1.3 and 0.7 $\mathrm{nm}$, respectively. These thicknesses were obtained by measuring an average of 10 different points around the nanoparticles, for about 30 nanoparticles at each concentration through the ImageJ software.

This organic layer is reduced by decreasing the concentration of diazonium salts. Concomitant decrease of aggregation of gold NPs was also noted for lower initial diazonium concentration. 
It should be noted that nanoparticles coated with two diazonium salts (AB and $D M B$ ) at a total concentration of $10^{-3} \mathrm{M}$ were very highly aggregated, and thus could not be characterized.

Figure 3e shows the organic layer around gold NPs with an average thickness of $1.8 \mathrm{~nm}$ for NPs/AB:DMB $10^{-4} \mathrm{M}$; a thinner $1 \mathrm{~nm}$ aryl layer was obtained for an initial diazonium concentration of $5 \times 10^{-5} \mathrm{M}$. In Figure $3 \mathrm{e}$, the thickness of organic layer is shown at a concentration of $10^{-4} \mathrm{M}$ of $\mathrm{AB}: \mathrm{DMB}$ salt mixture, which is more visible at higher concentrations of diazonium salts than the lower concentrations. In this regard, for two diazonium salts with a concentration of $5 \times 10^{-5} \mathrm{M}$, there is a better dispersion than in the case of $10^{-4} \mathrm{M}$ concentration.

\subsection{Thermal analysis (TGA/DTA)}

Thermal analysis TGA/DTA was performed for the grafted and co-grafted gold NPs under $\mathrm{O}_{2}$ to determine the mass of organic matter grafted to nanoparticles.

Table 1 shows the results of thermogravimetric analysis obtained with single and two diazonium-modified Au NPs.

The mass of organic layer at high concentrations of diazonium salt $A B$ is more than two salts $(\mathrm{AB} / \mathrm{DMB})$, while this trend is reverse for lower concentrations of $\mathrm{AB} / \mathrm{DMB}$ and the mass of organic layer in these two salts is more than single salts $\mathrm{AB}$.

There is a clear reduction in the mass of organic matter from $10^{-3} \mathrm{M}$ to $5 \times 10^{-5} \mathrm{M}$ diazonium salt concentrations, for both single and dual modifications. This decrease in the mass of organic matter occurs for single salts with a higher incidence than the use of salt mixtures.

Table 1. Analysis of the percentage of organic and mineral materials for nanoparticles modified with one and two diazonium salts in different concentrations by thermal analysis

\begin{tabular}{|c|c|c|}
\hline \multicolumn{3}{|c|}{ TGA/DTA } \\
\hline Products & $\%$ organic material & \% inorganic material \\
\hline NP/AB, $\mathbf{1 0}^{-3} \mathrm{M}$ & 6.9 & 93.1 \\
\hline $\mathrm{NP} / \mathrm{AB}, \mathbf{1 0}^{-4} \mathrm{M}$ & 3.2 & 96.8 \\
\hline $\mathrm{NP} / \mathrm{AB}, \mathbf{5} \times 10^{-5} \mathrm{M}$ & 2.5 & 97.5 \\
\hline NP/AB:DMB, $(1: 4) 10^{-3} \mathrm{M}$ & 4.7 & 95.3 \\
\hline NP/AB:DMB, $(1: 4) 10^{-4} M$ & 4.4 & 95.6 \\
\hline NP/AB:DMB, (1:4) $5 \times 10^{-5} \mathrm{M}$ & 4.11 & 95.89 \\
\hline
\end{tabular}

\subsection{XPS surface analysis}

Figure 4 displays Au4f doublet with $\mathrm{Au} 4 \mathrm{f}_{7 / 2}$ peak centered at $84 \mathrm{eV}$, characteristic of gold in the metallic state [41]. The ratio of the peaks $A u 4 f_{7 / 2}$ and $A u 4 f_{5 / 2}$ is $4: 3$ which testifies that there are no other gold oxidation states. 


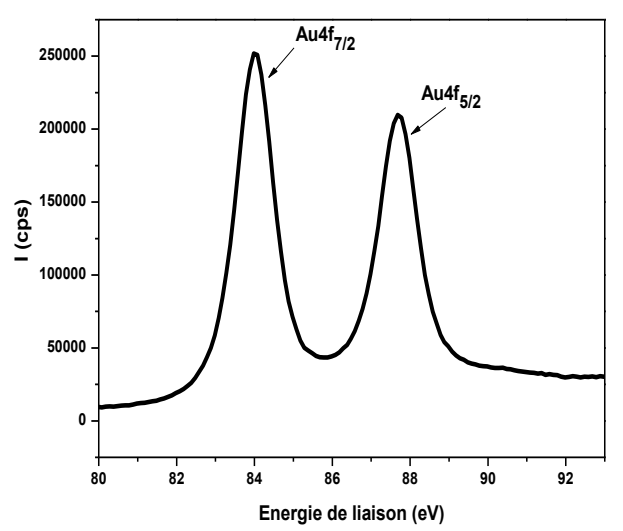

Figure 4. High resolution XP Au4f spectrum of pristine NPs powder after lyophilization.

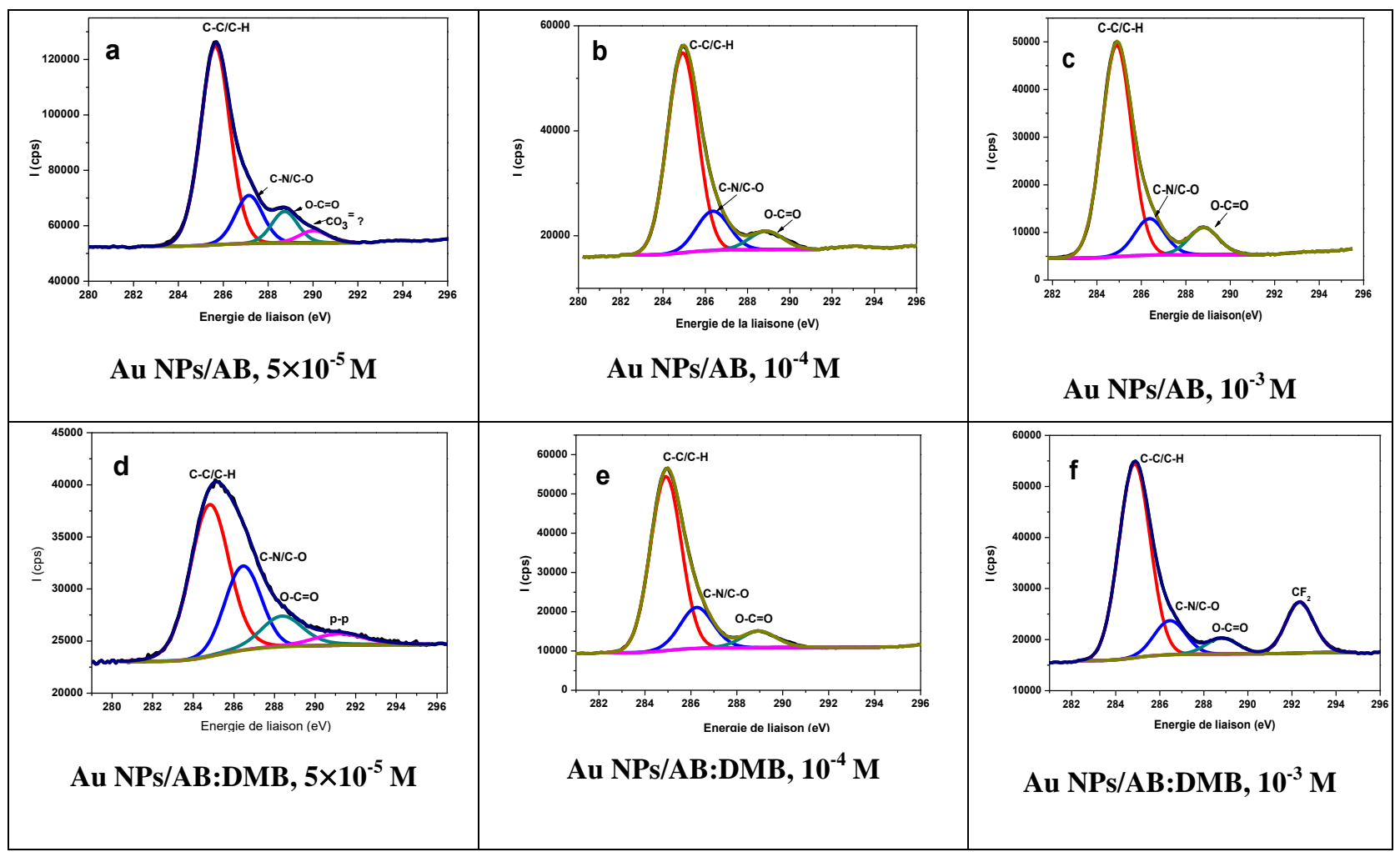

Figure 5. High-resolution $\mathrm{C}$ 1s spectra from grafted NPs/AB prepared with one diazonium salt in a) $10^{-3}$, b) $10^{-4}$ and c) $5 \times 10^{-5} \mathrm{M}$, and co-grafted NPs/AB:DMB prepared with two dizonium salts in d) $10^{-}$ ${ }^{3}$,e) $10^{-4}$ and f) $5 \times 10^{-5} \mathrm{M}$.

Figures 5a-f show peak fitted high resolution $\mathrm{C} 1 \mathrm{~s}$ narrow regions for gold NPs/AB and NPs/AB:DMB at different initial diazonium concentrations. Components centered at $284.8 \mathrm{eV}$ can be assigned to the reduced carbon $\mathrm{C}-\mathrm{C} / \mathrm{C}-\mathrm{H}$, at $286 \mathrm{eV}$ corresponds to the oxidized carbon atoms of $\mathrm{C}-\mathrm{O} / \mathrm{C}-\mathrm{N}$ and at $289 \mathrm{eV}$ assigned to the $\mathrm{O}-\mathrm{C}=\mathrm{O}$ group. Other detected species probably due to surface minor contamination are carbonates (see Figure 5a) and Teflon from the magnetic bar stirrer (see Figure 5f). All $\mathrm{C} 1 \mathrm{~s}$ regions invariably show the presence of the acidic group $\mathrm{COOH}$, which is related to diazonium salts $\mathrm{AB}$ grafted on gold nanoparticles. In this regard, at the concentrations of $10^{-4}$ and $5 \times 10^{-5} \mathrm{M}$ of two salts such as grafting with single salts show a decrease trend for the ratio of $\mathrm{C} / \mathrm{Au}$ by decreasing the 
concentration of diazonium salts; and thus, the number of aryl groups attached to the NP surface.

\subsection{Electrocatalytic oxidation of ethanol}

Figure 6 cyclic voltammetry shows no wave for GCE in the absence of any gold NPs and $\mathrm{EtOH}$ (voltammogram a-i). After adding $0.5 \mathrm{M} \mathrm{EtOH}$ this is observed an increase in the current intensity from $250 \mu \mathrm{A}$ (voltammogram Ai) to $420 \mu \mathrm{A}$ (voltammogram a-iii) at $1.497 \mathrm{~V}$ as well as formation of an oxidation wave at $1.35 \mathrm{~V}$ with the onset potential at $1.08 \mathrm{~V}$.

Voltammogram a-ii was obtained from modified electrode with gold NPs but in absence of ethanol. There is an oxidation wave at $1.4 \mathrm{~V}$ with an onset at $1.13 \mathrm{~V}$ that has almost the same current intensity as the oxidation wave of voltammogram (a-iii).

Voltammograms (a-iv and a-v) were obtained with the modified electrode in presence of 0.5 and $1 \mathrm{M}$ of ethanol, respectively. Adding $0.5 \mathrm{M}$ ethanol (voltammogram a-iv) resulted in an increase in current intensity from $340 \mu \mathrm{A}$ to $520 \mu \mathrm{A}$, and with an increase to $1 \mathrm{M}$ of ethanol in voltammogram (Av), the current intensity is $723 \mu \mathrm{A}$ at potential $1.497 \mathrm{~V}$. In these two voltammograms also appeared a new oxidation wave noted at $0.2 \mathrm{~V}[25,27,49]$ the current intensity of this wave increased with ethanol concentration.

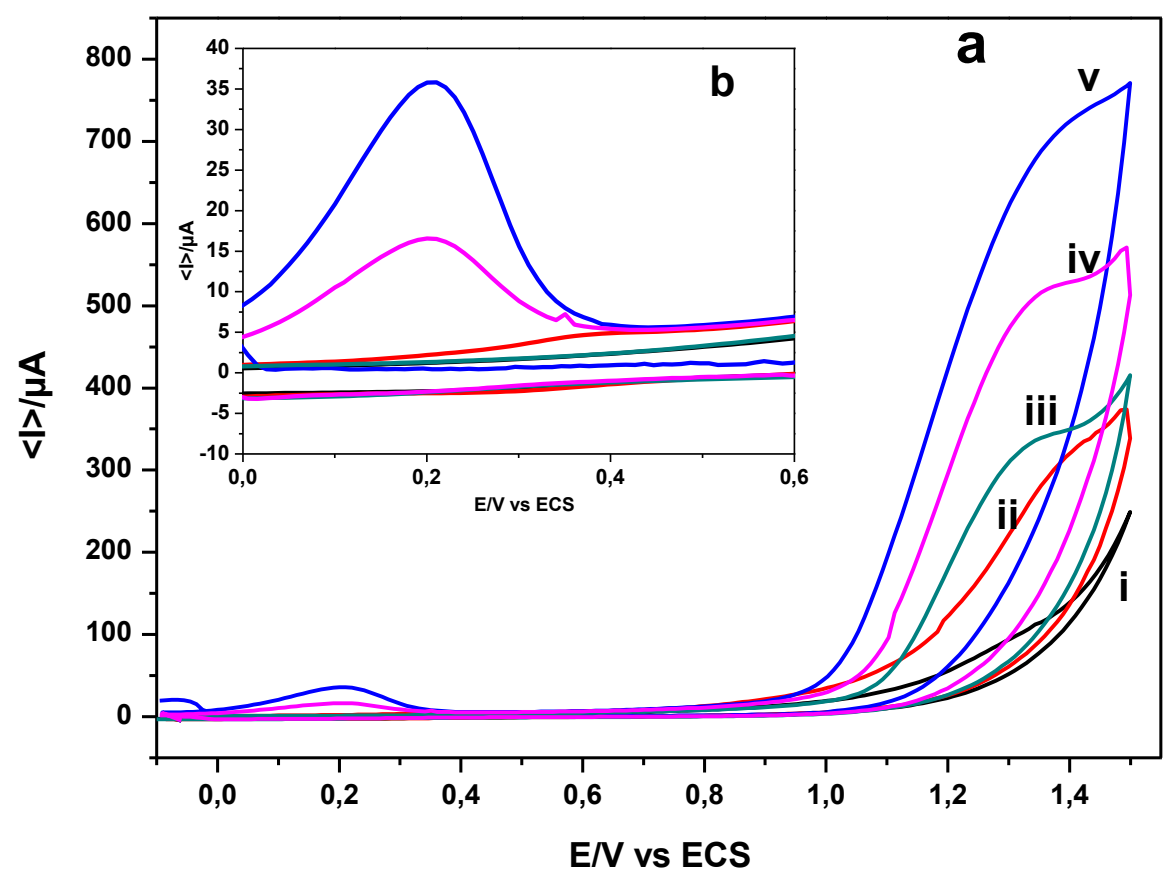

Figure 6. (a) Voltammograms of electro-oxidation of ethanol in a solution of $0.1 \mathrm{M} \mathrm{NaOH}, \mathrm{E}=-0.1$ / $1.5 \mathrm{~V}$ vs SCE, made on the glassy carbon electrode without NPs and without EtOH in black, with NPs and without EtOH in red, without NPs and with $0.5 \mathrm{M}$ of EtOH in green, with NPs and with $0.5 \mathrm{M}$ of EtOH in pink and with NPs and with $1 \mathrm{M}$ of EtOH in blue, (b) the zoom in 0/0.6V vs SCE. 
The voltammograms indicate that GCEs coated with the gold NPs are more active than the bare GCE for the oxidation of EtOH. Furthermore, there are 2 active oxidation potentials $(0.2$ $\mathrm{V}$ and $1.3 \mathrm{~V}$ vs. ECS) with these modified electrodes. Increase in the anodic current in the presence of ethanol shows that there is a catalytic activity in these potentials for electrooxidation of ethanol by gold NPs.
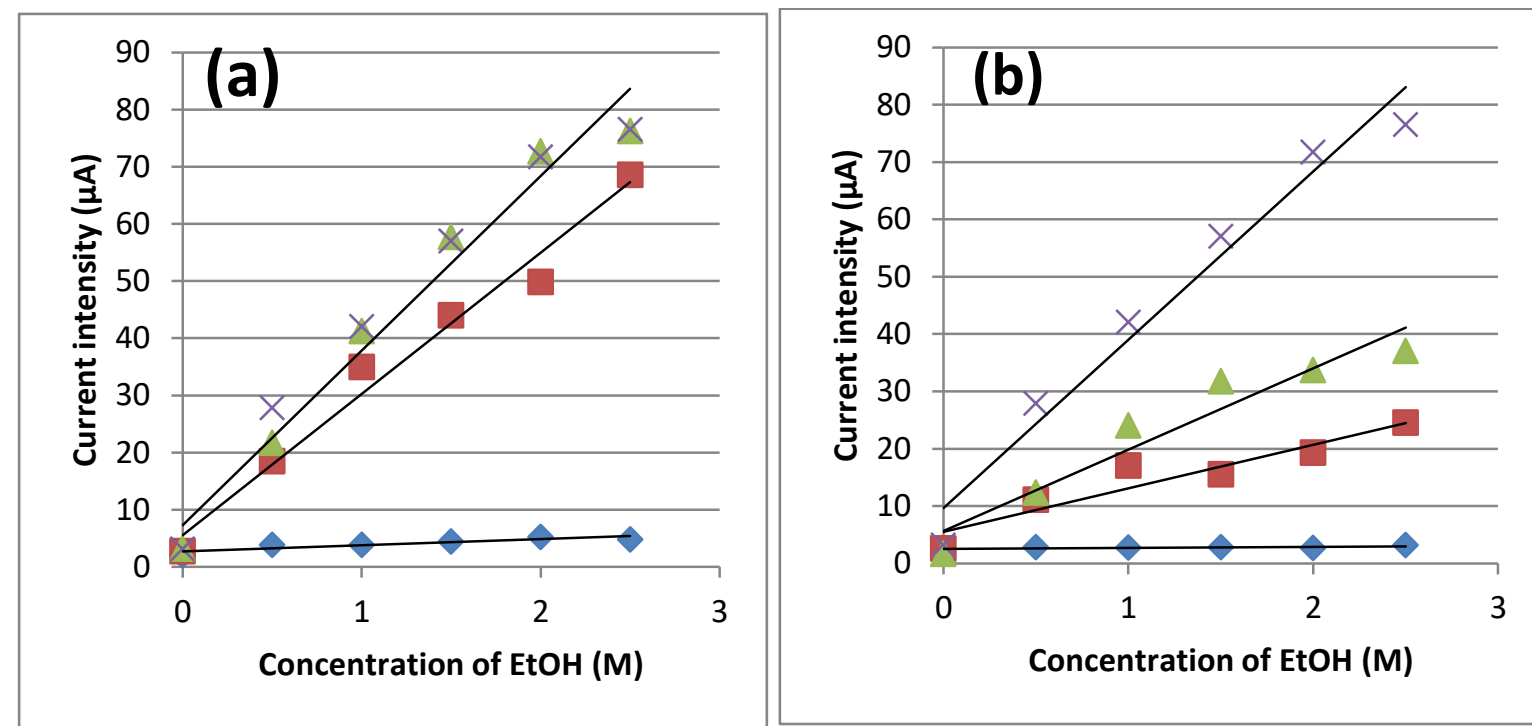

Figure 7. a) Comparison of curves of current intensity versus EtOH concentration for pristine gold NPs $(\mathbf{X})$ and grafted NPs/AB at $10^{-3}(\diamond), 10^{-4}(\mathbf{\square})$ and $5 \times 10^{-5} \mathrm{M}(\triangle)$, b) pristine gold NPs $(\mathbf{X})$ and cografted NPs/AB:DMB prepared with diazonium salts in different concentrations: $10^{-3}(\diamond), 10^{-4}(\square)$ and $5 \times 0^{-5} \mathrm{M}(\triangle)$.

Figure 7 shows current-vs-EtOH concentration linear plots for GCE electrodes coated with gold NPs/AB and gold NPs/AB:DMB. The current intensity corresponds to the peak of ethanol electro-oxidation noted at $0.2 \mathrm{~V}$ (see Figure 6). It is verified the current intensity of the oxidation wave as a function of the concentration of ethanol for gold NPs grafted with diazonium salt $\mathrm{AB}$ in different grafting concentration (Figure 7, a); these curves show that as the concentration of ethanol increases, the current intensity of the oxidation waves increases linearly. By increasing the initial diazonium salt concentration the current intensity of the oxidation wave decreases. For concentration $5 \times 10^{-5} \mathrm{M}$, the current intensity recorded with NPs/AB is very close to that obtained with ungrafted NPs.

For co-grafted NPs by AB and DMB, we compared their catalytic activity in different total concentrations of diazonium salts and ethanol, under the same conditions (Figure 7, b). From this comparison, it has been found that by increasing the concentration of the grafted salts on the NPs the catalytic efficiency decreases. Curves show two fold less catalytic efficiency for NPs/AB:DMB than NPs/AB when the diazonium initial concentration was $5 \times 10^{-5} \mathrm{M}$. It follows that dilution of the diazonium salt $\mathrm{AB}$ is more important than its mixture with $\mathrm{DMB}$, another way to obtain absolute low initial concentration. Most probably this is due to hydrophobic effects induced by the two methyl groups borne by DMB and which hamper access of ethanol to the nanocatalyst surface. Elsewhere, it was demonstrated that hydrophobic aryllayer can constitute barrier layer to redox probes [50, 51]. By analogy, it could be possible that ethanol molecules are likely to remain in the aqueous solution, away 
from a relatively hydrophobic aryl capping layer thus inducing a loss in the alcohol oxidation peak intensity.

\section{Conclusion}

This work reports on the design of novel aryl-modified gold nanoparticles using diazonium chemistry. The organically capped gold nanoparticles served as electrocatalysts of the ethanol oxidation. First, The Turkevich method was used to synthesize $20 \mathrm{~nm}$-sized gold nanoparticles that were readily arylated using diazonium salts. Then, individually, the diazonium salts $\mathrm{AB}$ and $\mathrm{DMB}$ were synthesized and served for the arylation of gold nanoparticles. TEM, XPS and TGA/DTA confirmed the formation of an aryl layer attached to nanoparticles with different thickness depending on the initial concentration of diazonium salts. Subsequently, the catalytic properties of the AuNPs were investigated for ethanol oxidation before and after grafting of diazonium salts. In this regard, by modifying gold nanoparticles with diazonium salts, the following results were obtained:

1- Maintaining the catalytic property of nanoparticles for ethanol oxidation after grafting the organic layer.

2- In comparing the catalytic properties of nanoparticles modified by a diazonium $\mathrm{AB}$ salt or two types of $\mathrm{AB}$ and $\mathrm{DMB}$ salts, it was found that NPs/AB at optimal concentration of diazonium salt $\left(5 \times 10^{-5} \mathrm{M}\right)$ there was no significant current intensity loss; however it was observed, this current intensity drop was greater in the use of two types of salt. This could be due to the decrease in the active surface of gold nanoparticles through further aggregation of these grafted nanoparticles because of the hydrophobicity of DMB salts.

3- Using the co-grafting of salts $\mathrm{AB}$ and $\mathrm{DMB}$ and determining the appropriate ratio and salts concentration, it was shown that surface engineering permitted to control the number of layers and also the ability to use other diazonium salts.

4- It is also noteworthy that using two diazonium salts $A B$ and $D M B$ in the same concentration $\left(5 \times 10^{-5} \mathrm{M}\right)$ led to a more homogeneous organic layer on the nanoparticles than using a single $\mathrm{AB}$ diazonium salt. This gives us better control over the homogeneity and thickness of the organic layer to keep working.

5- However, highest ethanol oxidation peak intensity was obtained for the lowest concentration of single $\mathrm{AB}$ diazonium concentration. Further dilution of $\mathrm{AB}$ by mixing with DMB resulted in les efficiency, likely due to barrier effect of the hydrophobic DMB methyl groups which hamper access of ethanol molecules to the nanocatalyst.

Ultimately, fine tuning of the dual arylation permitted to obtain even dispersion of the nanoparticles and access of the molecules to the catalytic cites at the NP surface without any decrease of the electrocatalytic property of nanoparticles. In this way, the dual arylated catalytic nanoparticles could be implemented in a more complete hybrid structure with specific, controlled properties and functionality. 


\section{Acknowledgements}

P. Mirzaei acknowledges MBA Water Treatment Chemicals Co. for the financial support of his PhD. AAM acknowledges the University of Sharjah support of competitive grants 160-2142-029-P and 150-2142-017-P, Organometallic Research Group grant RISE-046-2016, and Functionalized Nanomaterials Synthesis Lab grant 151-0039

Declaration of interest: the authors declare no conflict of interest.

\section{References}

[1] P. Gomez-Romero, Advanced Materials 13 (2001) 163-174.

[2] M.S. Saveleva, K. Eftekhari, A. Abalymov, T.E. Douglas, D. Volodkin, B.V. Parakhonskiy, A.G. Skirtach, Frontiers in chemistry 7 (2019) 179.

[3] M. Kalaj, K.C. Bentz, S. Ayala Jr, J.M. Palomba, K.S. Barcus, Y. Katayama, S.M. Cohen, Chemical reviews 120 (2020) 8267-8302.

[4] A.A. Ahmad, J.B. Marutheri Parambath, P.S. Postnikov, O. Guselnikova, M.M. Chehimi, M.R. Bruce, A.E. Bruce, A.A. Mohamed, Langmuir (2021).

[5] K. Kapat, Q.T. Shubhra, M. Zhou, S. Leeuwenburgh, Advanced Functional Materials 30 (2020) 1909045.

[6] F. Masse, M. Ouellette, G. Lamoureux, E. Boisselier, Medicinal research reviews 39 (2019) 302-327.

[7] S. Lee, E.J. Cha, K. Park, S.Y. Lee, J.K. Hong, I.C. Sun, S.Y. Kim, K. Choi, I.C. Kwon, K. Kim, Angewandte Chemie 120 (2008) 2846-2849.

[8] X. Zheng, S. Khaoulani, N. Ktari, M. Lo, A.M. Khalil, N.F. ENNOURI, C. Zerrouki, M.M. Chehimi, (2021).

[9] M.D. Hughes, Y.-J. Xu, P. Jenkins, P. McMorn, P. Landon, D.I. Enache, A.F. Carley, G.A. Attard, G.J. Hutchings, F. King, Nature 437 (2005) 1132-1135.

[10] B.N. Zope, D.D. Hibbitts, M. Neurock, R.J. Davis, Science 330 (2010) 74-78.

[11] J.M. Pingarrón, P. Yanez-Sedeno, A. González-Cortés, Electrochimica Acta 53 (2008) 58485866.

[12] P. Mirzaei, S. Bastide, A. Aghajani, J. Bourgon, E. Leroy, J. Zhang, Y. Snoussi, A. Bensghaier, O. Hamouma, M.M. Chehimi, Langmuir 35 (2019) 14428-14436.

[13] M. Lo, N. Ktari, D. Gningue-Sall, A. Madani, S.E. Aaron, J.-J. Aaron, Z. Mekhalif, J. Delhalle, M.M. Chehimi, Emergent Materials (2020) 1-25.

[14] F. Mousli, A.M. Khalil, F. Maurel, A. Kadri, M.M. Chehimi, Cellulose 27 (2020) 7823-7846.

[15] M. Bharti, A. Singh, S. Samanta, D. Aswal, Progress in Materials Science 93 (2018) 270-310.

[16] Y. Duan, C. Ezquerro, E. Serrano, E. Lalinde, J. García-Martínez, J.R. Berenguer, R.D. Costa, Advanced Functional Materials 30 (2020) 2005401.

[17] A.A. Ahmad, A.H. Alawadhi, J. Park, H.E. Abdou, A.A. Mohamed, Forensic Chemistry 13 (2019) 100144.

[18] C. Sanchez, B. Julián, P. Belleville, M. Popall, Journal of Materials Chemistry 15 (2005) 3559-3592.

[19] B.V.K. Naidu, J.S. Park, S.C. Kim, S.-M. Park, E.-J. Lee, K.-J. Yoon, S.J. Lee, J.W. Lee, Y.S. Gal, S.-H. Jin, Solar Energy Materials and Solar Cells 92 (2008) 397-401.

[20] S. Sista, M.H. Park, Z. Hong, Y. Wu, J. Hou, W.L. Kwan, G. Li, Y. Yang, Advanced materials 22 (2010) 380-383.

[21] K. Jlassi, S. Zavahir, P. Kasak, I. Krupa, A.A. Mohamed, M.M. Chehimi, Energy conversion and management 168 (2018) 170-177.

[22] Q. Lenne, Y.R. Leroux, C. Lagrost, ChemElectroChem 7 (2020) 2345-2363.

[23] J. Mathiyarasu, S. Senthilkumar, K. Phani, V. Yegnaraman, Materials Letters 62 (2008) 571 -

573.

[24] F. Mirkhalaf, J.E. Graves, Chemical Papers 66 (2012) 472-483. 
[25] R.K. Pandey, V. Lakshminarayanan, Applied Catalysis B: Environmental 125 (2012) 271-281. [26] J. Monzó, Y. Malewski, F.J. Vidal-Iglesias, J. Solla-Gullon, P. Rodriguez, ChemElectroChem 2 (2015) 958-962.

[27] C. Wang, K. Zhang, H. Xu, Y. Du, M.C. Goh, Journal of colloid and interface science 541 (2019) 258-268.

[28] L. Liu, H. Li, Y. Tan, X. Chen, R. Lin, W. Yang, C. Huang, S. Wang, X. Wang, X.Y. Liu, Catalysts 10 (2020) 107.

[29] E. Contreras, C. Palacios, B. Huerta, S. Ahn, O. Vazquez-Mena, R.D. Cadena-Nava, G. Alonso-Nunez, O.E. Contreras, M.T. Oropeza-Guzmán, J.M. Romo-Herrera, ACS Applied Energy Materials 3 (2020) 8755-8764.

[30] C. Wang, F. Jiang, R. Yue, H. Wang, Y. Du, Journal of Solid State Electrochemistry 18 (2014) 515-522.

[31] R. Yue, H. Wang, D. Bin, J. Xu, Y. Du, W. Lu, J. Guo, Journal of Materials Chemistry A 3 (2015) 1077-1088.

[32] L. Laurentius, S.R. Stoyanov, S. Gusarov, A. Kovalenko, R. Du, G.P. Lopinski, M.T. McDermott, ACS nano 5 (2011) 4219-4227.

[33] F. Mirkhalaf, J. Paprotny, D.J. Schiffrin, Journal of the American Chemical Society 128 (2006) 7400-7401.

[34] M. Lo, A.K. Diaw, D. Gningue-Sall, J.-J. Aaron, M.A. Oturan, M.M. Chehimi, Electrochemistry Communications 77 (2017) 14-18.

[35] M. Lo, M. Seydou, A. Bensghaïer, R. Pires, D. Gningue-Sall, J.-J. Aaron, Z. Mekhalif, J. Delhalle, M.M. Chehimi, Sensors 20 (2020) 580.

[36] A.A. Mohamed, Z. Salmi, S.A. Dahoumane, A. Mekki, B. Carbonnier, M.M. Chehimi, Advances in colloid and interface science 225 (2015) 16-36.

[37] G. Liu, E. Luais, J.J. Gooding, Langmuir 27 (2011) 4176-4183.

[38] F. Mirkhalaf, D.J. Schiffrin, Langmuir 26 (2010) 14995-15001.

[39] S. Gam-Derouich, S. Mahouche-Chergui, S. Truong, D.B. Hassen-Chehimi, M.M. Chehimi, Polymer 52 (2011) 4463-4470.

[40] S. Belbekhouche, S.I. Kebe, S. Mahouche-Chergui, M. Guerrouache, B. Carbonnier, M. Jaziri, M.M. Chehimi, Colloids and Surfaces A: Physicochemical and Engineering Aspects 529 (2017) 541549.

[41] A. Bensghaïer, V. Bhullar, N. Kaur, M. Lo, M. Bdiri, A. Mahajan, M.M. Chehimi, Emergent Materials 4 (2021) 515-524.

[42] C. Combellas, D.-e. Jiang, F. Kanoufi, J. Pinson, F.I. Podvorica, Langmuir 25 (2009) 286-293.

[43] C. Jiang, S.M. Silva, S. Fan, Y. Wu, M.T. Alam, G. Liu, J.J. Gooding, Journal of Electroanalytical Chemistry 785 (2017) 265-278.

[44] J. Turkevich, P.C. Stevenson, J. Hillier, Discussions of the Faraday Society 11 (1951) 55-75.

[45] G. Liu, Y. Zhang, M. Qi, F. Chen, Analytical Methods 7 (2015) 5619-5626.

[46] R. Ahmad, L. Boubekeur-Lecaque, M. Nguyen, S.p. Lau-Truong, A. Lamouri, P. Decorse, A. Galtayries, J. Pinson, N. Felidj, C. Mangeney, The Journal of Physical Chemistry C 118 (2014) 1909819105.

[47] R. Ahmad, N. Félidj, L. Boubekeur-Lecaque, S. Lau-Truong, S. Gam-Derouich, P. Decorse, A. Lamouri, C. Mangeney, Chemical Communications 51 (2015) 9678-9681.

[48] R.G. Acres, V. Feyer, N. Tsud, E. Carlino, K.C. Prince, The Journal of Physical Chemistry C 118 (2014) 10481-10487.

[49] Y. Zhang, J.-G. Wang, X. Yu, D.R. Baer, Y. Zhao, L. Mao, F. Wang, Z. Zhu, ACS Energy Letters 4 (2018) 215-221.

[50] A.J. Downard, M.J. Prince, Langmuir 17 (2001) 5581-5586.

[51] M. Lo, R. Pires, K. Diaw, D. Gningue-Sall, M.A. Oturan, J.-J. Aaron, M.M. Chehimi, Surfaces 1 (2018) 43-58. 